Pol. Con. (Edición núm. 5) Vol. 2, No 3

Marzo 2017, pp. 207-2016

ISSN: 2550 - $682 \mathrm{X}$

DOI: 10.23857/pc.v2i3.201

Recepción: 20 / 01 / 2017

Aceptación: 20 / 02 / 2017

Publicación: 15 / 03 / 2017

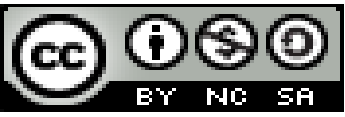

Ciencias Médicas

Reporte de Caso

\title{
Embarazo ectópico a propósito de dos casos
}

Ectopic pregnancy in relation to cases

Gravidez ectópica em relação aos casos

María Auxiliadora Calero-Zea ${ }^{\mathrm{I}}$ _maria.caleroz@ug.edu.ec

Blanca Elena Andrade-Burgos II blanca.andradeb@ug.edu.ec

Amando Sánchez-Alcívar ${ }^{\text {III }}$ amando.sancheza@ug.edu.ec

Julio Ramón Villacrés-Pastor ${ }^{\text {IV }}$ julio.villacresp@ug.edu.ec

Correspondencia:_maria.caleroz@ug.edu.ec

\footnotetext{
${ }^{\text {I } M e ́ d i c o, ~ U n i v e r s i d a d ~ d e ~ G u a y a q u i l, ~ G u a y a q u i l, ~ E c u a d o r . ~}$

${ }^{\text {II } M e ́ d i c o, ~ U n i v e r s i d a d ~ d e ~ G u a y a q u i l, ~ G u a y a q u i l, ~ E c u a d o r . ~}$

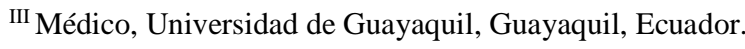

${ }^{\text {IV }}$ Médico, Universidad de Guayaquil, Guayaquil, Ecuador.
} 


\section{Resumen}

En la mayoría de los embarazos, el óvulo fecundado viaja a través de las trompas de Falopio hacia la matriz (útero). Si el movimiento de este óvulo a través de las trompas se bloquea o se retrasa puede llevar a que se presente un embarazo ectópico. Los factores que pueden causar este problema abarcan desde defecto congénito en las trompas, cicatrización de una apendicitis perforada, endometriosis, antecedente de embarazo ectópico y los factores predisponentes como la edad (mayor de 35 años), embarazo + DIU, salpingectomía, recanalización tubárica, tratamientos de infertilidad, etc.

Algunas veces, se desconoce la causa. Las hormonas pueden jugar un papel fundamental. El sitio más común para un embarazo ectópico está dentro de una de las dos trompas de Falopio. En raras ocasiones, los embarazos ectópicos pueden presentarse en los ovarios, el abdomen o el cuello uterino, puede ocurrir incluso si se usa anticonceptivos, se puede sentir síntomas tempranos de embarazo, como sensibilidad mamaria o náuseas. Otros síntomas pueden abarcar: Sangrado vaginal anormal, lumbago, cólico leve en un lado de la pelvis, ausencia de menstruación, dolor en la parte inferior del abdomen o en la zona de la pelvis, si la zona alrededor del embarazo anormal presenta ruptura y sangra, los síntomas pueden empeorar. Estos pueden abarcar: Desmayo o sensación de desmayo,presión intensa en el recto, presión arterial baja, dolor en el área del hombro, dolor agudo, súbito e intenso en la parte inferior del abdomen, pudiendo llegar al shock hipovolémico y muerte, de ahí la gran importancia de su diagnóstico precoz. Se publica el estudio sobre dos casos de la Clinica central de Aprofe en el que observamos en el primero embarazo a nivel de la trompa derecha y el segundo a nivel del ligamento

Palabras clave: Embarazo ectópico; anticonceptivos; hormonas; trompas de Falopio. 


\section{Abstract}

In most of the pregnancies, the fertilized ovum travels across the Fallopian tubes towards the counterfoil (womb). If the movement of this ovum across the tubes is blocked or is slow, it can end in an ectopic pregnancy. The factors that can cause this problem goes from congenital defect in the tubes, healing of a perforated appendicitis, endometriosis, history of ectopic pregnancies to predisposing factors like the age (more than 35 years old), pregnancy + IUD, salpingectomy, tubaric recanalization, infertility treatments, etc.

Sometimes, the cause is unknown. The hormones play a fundamental role. The most common place for an ectopic pregnancy is inside one of the two Fallopian tubes. In rare occasions, the ectopic pregnancies can appear in the ovaries, the abdomen or the neck of the womb, it can happen even if contraceptive methods are used; it is possible to feel early pregnancy symptoms, like mammary sensibility or morning sickness. Other symptoms can include abnormal vaginal bleeding, back pain, non-severe colic in a pelvis' side, amenorrhea, pain in the lower part of the abdomen or in the area of the pelvis, if the area surrounding the abnormal pregnancy presents ruptures and bleeding, the symptoms can deteriorate. They can include these: Faint or sensation of faint, intense pressure in the rectum, low blood pressure, shoulder pain, acute, sudden and intense pain in the lower part of the abdomen, which can end up in hypovolemic shock and death hence the big importance of its aerly diagnosis. The current study shows two cases of the central Clinic of Aprofe in which we observe in the first case an ectopic pregnancy at level of the right tube and the second case at level of the ligament.

Key words: Ectopic pregnancy; Contraceptives; Hormonal; Fallopian tube. 


\section{Resumo}

$\mathrm{Na}$ maioria das gravidezes, o óvulo fertilizado viaja através das trompas de Falópio para o contramestre (útero). Se o movimento deste óvulo através dos tubos estiver bloqueado ou lento, ele pode acabar em uma gravidez ectópica. Os fatores que podem causar esse problema vão do defeito congênito nos tubos, cicatrização de uma apendicite perfurada, endometriose, história de gravidez ectópica em fatores predisponentes como idade (mais de 35 anos), gravidez + DIU, salpingectomia, recanalização tubarica, Tratamentos de infertilidade, etc.

Às vezes, a causa é desconhecida. Os hormônios desempenham um papel fundamental. O lugar mais comum para uma gravidez ectópica está dentro de uma das duas trompas de Falópio. Em raras ocasiões, as gravidezes ectópicas podem aparecer nos ovários, no abdômen ou no pescoço do útero, pode ocorrer mesmo que se utilizem métodos contraceptivos; É possível sentir sintomas precoce da gravidez, como a sensibilidade mamária ou a doença da manhã. Outros sintomas podem incluir sangramento vaginal anormal, dor nas costas, cólica não grave no lado da pelve, amenorréia, dor na parte inferior do abdômen ou na área da pelve, se a área que envolve a gravidez anormal apresenta rupturas e sangramento, Os sintomas podem se deteriorar. Eles podem incluir: fraco ou sensação de pressão fraca e intensa no reto, baixa pressão arterial, dor no ombro, dor aguda, repentina e intensa na parte inferior do abdômen, que pode acabar em choque hipovolêmico e morte, portanto, o grande Importância do seu diagnóstico aéreo. O estudo atual mostra dois casos da clínica central de Aprofe em que observamos no primeiro caso uma gravidez ectópica no nível do tubo direito e o segundo caso no nível do ligamento.

Palavras chave: Gravidez ectópica; contraceptivos; hormonal; trompas de Falópio. 


\section{Introducción.}

Se revisaron diferentes autores de la literatura mundial, incluyendo investigadores cubanos del tema ${ }^{1-10}$ quienes admiten que "existe un aumento de la frecuencia del embarazo ectópico en el mundo, incluyendo a Cuba", hasta 5 veces más cuando se compara con la década del 70.

Novak ${ }^{11}$ destaca en 1970, una frecuencia de EE de 1:300-200 gestaciones. En el libro de Patología estructural de Robbins ${ }^{12}$ se señala en 1983 que se eleva la frecuencia de EE a 1:100-150 gestaciones, con una mortalidad de 1:400 casos, mientras que en el 2004, Rigol $^{13}$ expone que la frecuencia del EE, representa un 2,2 \% del número total de los nacimientos y el 1,7 \% de las gestaciones conocidas.

Se considera que esta afección adquiere gran importancia, ya que mundialmente "hasta el 2004, al embarazo ectópico, se debe entre el 5 y el $12 \%$ de las muertes maternas, lo que constituye un problema no resuelto" y así lo destacan las organizaciones mundiales de salud, la OMS y la UNICEF. ${ }^{14}$

En Cuba, las cifras de muerte materna que existían en la década 1950, se han reducido en un $78,3 \%$, comparada con el año 2004, pero entre las causas que persisten y que han aumentado, está el EE complicado. ${ }^{15}$ La tasa de muerte materna directa por EE en el país se eleva progresivamente en los últimos años, alcanzando en el año 2000, la cifra de 3,5 por 100000 nacidos vivos y asciende en el 2003 a 5,8 por 100000 nacidos vivos, según el anuario estadístico nacional. ${ }^{16}$

La presente investigación pretender realizar revisión sobre el embarazo ectópico (EE), puede parecer de entrada una insistencia innecesaria en asuntos altamente conocidos, sin embargo, el incremento de su incidencia afirma lo contrario. 
María Auxiliadora Calero-Zea; Blanca Elena Andrade-Burgos; Amando Sánchez-Alcívar; Julio Ramón Villacrés-

Pastor

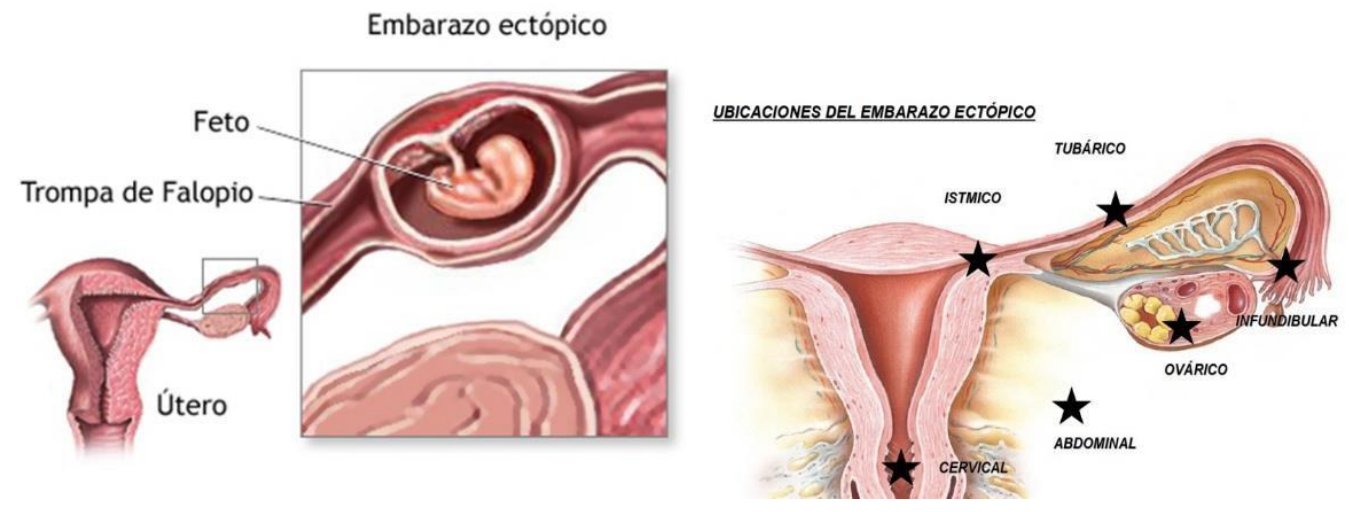

Fig 1.- a) esquema de localización tubárica; b) Posibles ubicaciones de embarazos ectópicos

\section{Materiales y Métodos}

Se realiza una prueba de embarazo y una ecografía vaginal en los dos casos.

La HCG es una hormona que normalmente se produce durante el embarazo. El chequeo de los niveles de esta hormona en la sangre puede diagnosticar el embarazo. Si el nivel sanguíneo de GCH no se está elevando lo suficientemente rápido, el médico puede sospechar de un embarazo ectópico. En ambos casos estuvo positiva

En el caso 1 existía liquido libre en Douglas e imagen de pseudosaco en relación a embarazo tubárico derecho con aumento de la vascularización y dolor a la compresión 


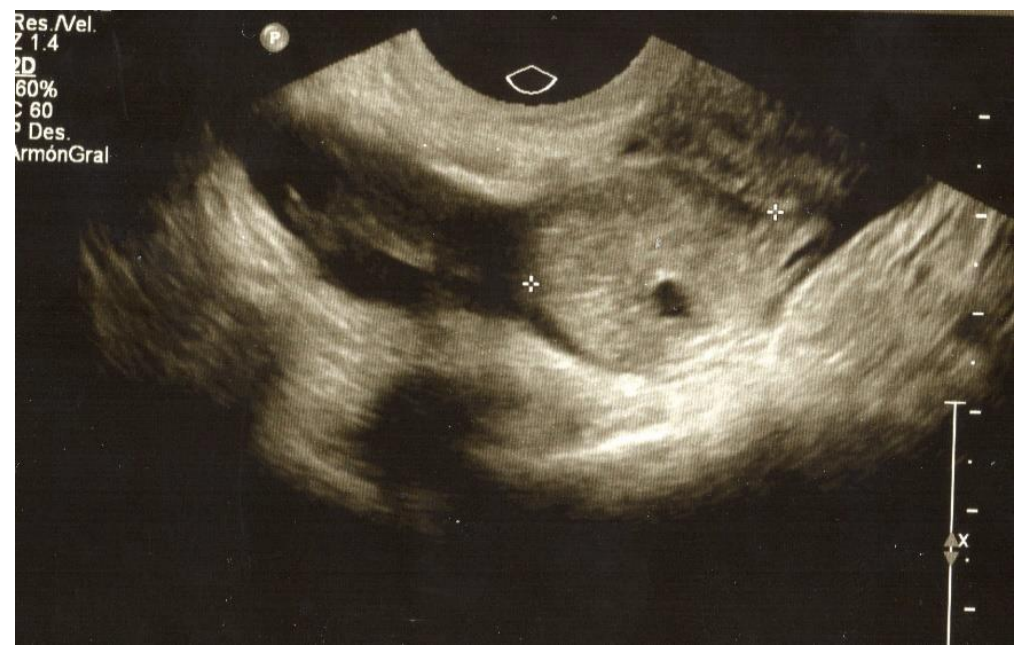

Fig 3.- Imagen de pseudosaco con líquido en Douglas derecho y aumento vascular, reacción decidual en endometrio

En el caso 2 se visualizaba saco gestacional por debajo de trompa, paralelo al ovario izquierdo en relación a embarazo ectópico a nivel del ligamento encontrándose embrión vivo con latidos embriocárdicos.

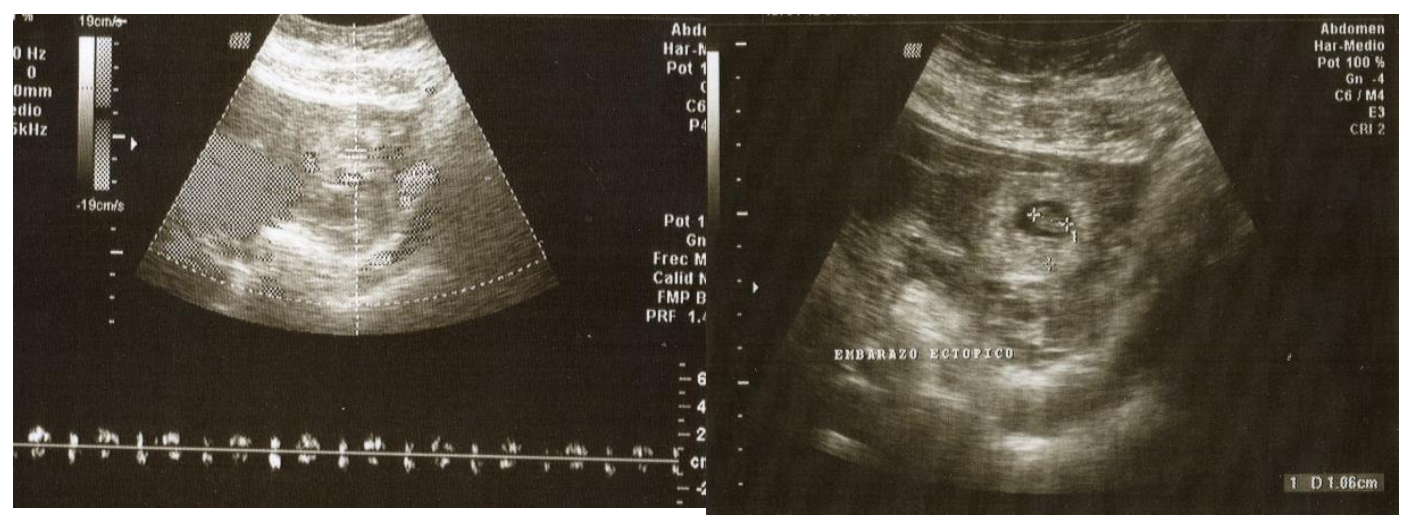

Fig4.- Saco gestacional con embrión vivo de 10mm de Lcr en relación a 5-6 semanas por u.s Fc $120 X^{*}$ 


\section{Tratamiento.}

El embarazo ectópico es potencialmente mortal y no puede continuar hasta el nacimiento (a término). Ambos casos fueron a cirugía en el caso 1 se realizó salpingectomía derecha y en el caso 2 fue interligamentario pudiendo salvarse la trompa izquierda .

\section{Discusión y conclusión}

La incidencia de embarazo ectópico ha aumentado en los últimos 30 años y aunque ha disminuido su morbimortalidad, es la primera causa actual de mortalidad en el primer trimestre del embarazo. Su sospecha precoz es importante, ante toda mujer en edad fértil y con factor de riesgo indicativos de una gestación extrauterina.

Una de cada tres mujeres que ha tenido un embarazo ectópico puede tener un bebé más adelante. Es más probable que en ellas se presente otro embarazo ectópico. Algunas mujeres no vuelven a quedar embarazadas de nuevo.

La probabilidad de un embarazo exitoso después de un embarazo ectópico depende de varios factores como la edad de la mujer, si es multípara o por el contrario el ectópico fue su primer embarazo, múltiples factores que van a ser vitales para el futuro embarazo .

Es de suma importancia el abordaje precoz para evitar la ruptura e intentar salvar la trompa de Falopio para dar mayor oportunidad a estas mujeres a tener embarazos subsiguientes,

El ultrasonido vaginal en manos de un operados entrenado y las determinaciones seriadas de gonadotropina coriónica humana $\beta$ ( $\beta$-hCG) son actualmente, los métodos más usados para el 
diagnóstico de embarazo ectópico. Esta combinación brinda una sensibilidad de $96 \%$ y una especificidad de $97 \%$ para realizar el diagnóstico.

\section{Bibliografía.}

1. Botella Llusiá J, Clavero Núñez LA. Tratado de Ginecología. 14tª ed. Madrid: Editorial Díaz Santos; 1993.

2. Williamns. Tratado de Obstetrica. $21^{\text {ra }}$ ed. La Habana: Editorial Ciencias Médicas; 2007.

3. Silva Solovera S. Revisión bibliográfica de Embarazo Ectópico del 1992-2003. Servicio de Obst y Ginec Hosp Dr. Sótero del Río, Stgo de Chile. 2004. [citado 9 Sep 2004]. Disponible en: http://www.buenastareas.com/ensayos/Embarazo-Ectopico/631999.html

4. Durán Rodríguez FJ. ¿Se diagnostica siempre a tiempo un embarazo ectópico? Socuéllamos (C Real) Rev Sem G. 2003;(55):424-6.

5. Martínez J, Basáez E, Rodríguez P. Embarazo ectópico. Chile RMS. 2000;3(12).

6. Matos Quiala HA, Rodríguez Lara O, Estrada Sánchez G, Martínez Enfedaque M, Saap Nuño A, Osorio Rodríguez M, et al. Caracterización de embarazo Ectópico. Rev inf cient. 2008;58(2):1-9.

7. Cutié Bressler ML, Figueroa Mendoza M, Lestayo Dorta C, Cubero Menéndez O. Embarazo Ectópico. Su comportamiento en los años 1989-1999. Rev Cub Obstet Ginecol. 2001;27(1): 5-11.

8. Casanova Carrillo C. Epidemiología del embarazo ectópico en un hospital ginecoobstétrico. Rev Cub Enfermer [serie e Internet]. 2003 [citado 17 May 2008];19(3). Disponible en: $\underline{\text { http://scielo.sld.cu/scielo.php?script=sci_arttext\&pid=S0864-03192003000300003\&lng=es\&nrm=iso }}$

9. Olalde Cancio R. Embarazo Ectópico: factores relacionados y otras consideraciones. Medicentro. $2004 ; 8(4)$.

10. Capote Arce R, Carrillo Bermúdez L, Guzmán Parrado R, Varona J, Mohamed Abdelaziz M. Embarazo ectópico cervical: presentación de un caso. Rev Cubana Obstet Ginecol [serie en Internet]. 2007 Abr [citado 16 Nov 2010];33(1): Disponible en: http://scielo.sld.cu/scielo.php?script=sci arttext\&pid=S0138$\underline{600 \times 2007000100002 \& \operatorname{lng}=e s}$

11. Novak ER, Seegar Jones G, Jones HW. Tratado de Ginecología. Madrid: Ed Interam Mc Graw-Hill; 1991.

12. Cotran RS. Patología estructural y funcional de Robbins. Madrid: Ed. Mc Graw Hill Interam; 2000.

13. Rigol Ricardo O. Obstetricia y Ginecología. $2^{\text {da }}$ ed. La Habana: Editorial Ciencias Médicas; 2004.

14. Maternal Mortality in 2000. Estimates Developed by WHO, UNICEF and UNFPA. Department of Reproductive Health and Research. Geneva: World Health Organization; 2004. Disponible en: http://www.who.int/features/qa/12/es/index.Html 
María Auxiliadora Calero-Zea; Blanca Elena Andrade-Burgos; Amando Sánchez-Alcívar; Julio Ramón Villacrés-

Pastor

15. Cabezas Cruz E. Evolución de la mortalidad materna en Cuba. Rev Cubana Sal Públ [serie en Internet]. 2006 [citado

17

May

2008];32(1).

Disponible

en: http://scielo.sld.cu/scielo.php?script=sci arttext\&pid=S0864-34662006000100005\&lng=es\&nrm=iso 